\title{
A Sufficient Condition For Computing N-Finger Force-Closure Grasps of 3D Objects
}

\author{
Sahar EL-Khoury \\ LISIF - EA2385 \\ Universite Pierre et Marie Curie - Paris6 \\ Paris, France \\ s.khoury@lisif.jussieu.fr
}

\author{
Anis Sahbani \\ LISIF - EA2385 \\ Universite Pierre et Marie Curie - Paris6 \\ Paris, France \\ anis.sahbani@upmc.fr
}

\begin{abstract}
We address the problem of computing n-finger force-closure grasps of 3D objects. As $3 D$ force-closure grasps involve $6 D$ wrench space, we use Plücker coordinates and Grassmann algebra, to demonstrate that wrenches associated to any three non-aligned contact points of $3 D$ objects form a basis of the $6 D$ wrench space. Thus, given non-aligned locations of $n-1$ fingers, a $6 D$ basis can be extracted form their wrenches. This permits the formulation of a fast and simple sufficient forceclosure test. The problem is transformed to searching for a set of locations of the $n t h$ finger which wrenches can be uniquely expressed as a strictly negative linear combination of the $6 D$ basis. We have implemented the algorithm and confirmed its efficiency by comparing it to the classical convex-hull method [21].
\end{abstract}

Index Terms-Force-closure grasps, Grassmann algebra.

\section{INTRODUCTION}

Many researchers are interested in developing humanoid robots to help people in their daily life. Such robots should be autonomous and able to interact with objects around them. Grasping is the central action of object manipulation. A grasp should satisfy several conditions such as stability, collisionavoidance, task compatibility etc. This paper considers generating stable grasps.

The stability of a grasp is characterized by force-closure property [1], under which arbitrary forces and torques exerted on the grasped object can be balanced by the contact forces applied by the fingers. We address the problem of computing $\mathrm{n}$-finger force-closure grasps of 3D objects. We assume hardfinger point contacts with friction.

Salisbury and Roth [2] have proved that a necessary and sufficient condition for force closure is that the primitive contact wrenches resulted by contact forces at the contact points positively span the entire wrench space. This condition is equivalent to that the origin of the wrench space lies strictly inside the convex hull of the primitive contact wrenches [5], [6]. Based on the above necessary and sufficient conditions, various force-closure tests were proposed by Nguyen [12] and Mishra et al. [4].

In the past few years, several force-closure tests were also proposed. By introducing the polyhedral approximation of the non linear friction cone, Y.H. Liu [3] demonstrates that the problem of querying whether the origin lies inside the convex hull is equivalent to a ray shooting problem. Zhu and
Wang [20] developed a numerical force closure test based on the concept of the Q-distance. All these methods require considerable computation time.

Heuristic approaches are a way to improve performance. Borst et al. [18] showed that with a strategy to randomly generate grasps and filter them with simple heuristics, the calculation of force-closure grasps can be done very fast. The heuristic of Niparnan and Sudsang [19] relies on a necessary but not sufficient condition of force-closure. It works as a filter that reports a fault positive but not a fault negative force-closure grasps. We propose a sufficient but not necessary method to compute force-closure grasps of 3D objects. Our approach works with general 3D objects and with any number $n \geq 4$ of contacts. The locations, normal directions and friction coefficient of these contacts are known.

The rest of the paper is organized as follows. Section 2 introduces notations and theorems used in the following of the paper. The proposed force-closure test is presented in section 3. Section 4 details the approach to compute $n$-finger force-closure grasps. Sections 5 shows experimental results and a comparison with the classical convex-hull method. Section 6 concludes.

\section{PRELIMINARIES}

Our goal is to reduce the overall time to compute possible grasping points of the $n$-finger force-closure grasps. With a change of mathematical representation, we prove that wrenches, associated to any three non-aligned contact points of $3 \mathrm{D}$ objects form a basis of the $6 D$ wrench space. This result induce the formulation of a simple sufficient condition of force-closure frictional grasps.

This section presents definitions, theorems and notations necessary for force-closure test elaboration.

\section{A. Grasp Preliminaries}

A grasp map or a wrench matrix is crucial to determine if a grasp has force-closure. This paragraph introduces all elements to compute this matrix.

Definition 1: A grasp is a set of contacts. 
Definition 2: A contact is a location where a finger meets the object surface. Information about contact type and local object surface are required. We assume a point contact model with coulomb friction.

Definition 3: A grasp force $f_{i}$ is a force applied by each finger to the object.

To ensure nonslipping at the contact point, the grasp force $f_{i}$ must satisfy coulomb's law [22], [23]:

$$
f_{i x}^{2}+f_{i y}^{2} \leq \mu^{2} f_{i z}^{2}
$$

where $\left(f_{i x}{ }^{2}, f_{i y}{ }^{2}, f_{i z}{ }^{2}\right)$ denotes $x, y, z$ components of the grasp force $f_{i}$ in the object coordinate frame and $\mu$ the friction coefficient.

Definition 4: The non linear constraint in (1) geometrically defines a cone called friction cone.

To simplify the problem, we linearize the friction cone by a polyhedral convex cone with $m$ sides.

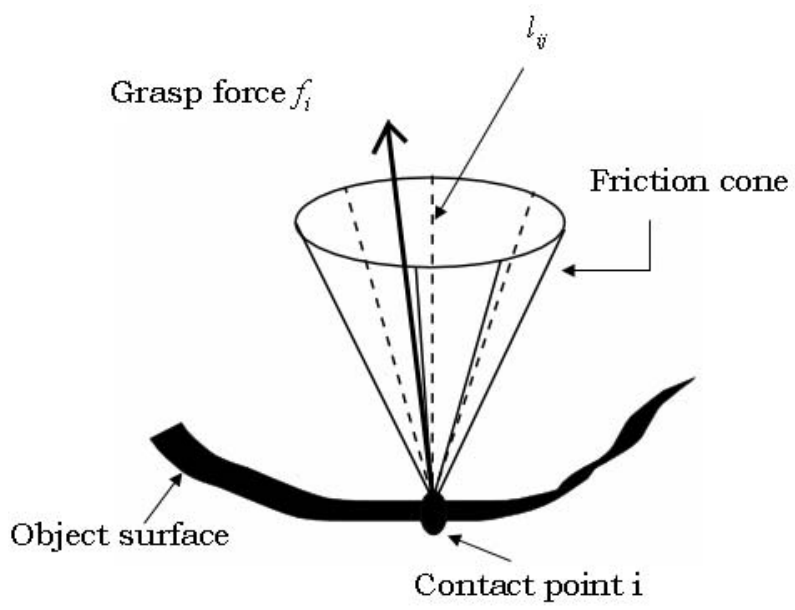

Fig. 1. The grasp force $f_{i}$ in a linearized friction cone

Under this approximation, the grasp force can be represented as:

$$
f_{i}=\sum_{i=1}^{m} \lambda_{i j} l_{i j}, \quad \lambda_{i j} \geq 0
$$

where $l_{i j}$ represents the $\mathrm{j}$-th edge vector of the polyhedral convex cone. Coefficients $\lambda_{i j}$ are non negative constants.

Definition 5: A wrench, $\underline{w}_{i}$, is the combination of the force and torque corresponding to the grasp force $f_{i}$.

$$
\underline{w}_{i}=\left(\begin{array}{c}
f_{i} \\
\tau_{i}
\end{array}\right)=\left(\begin{array}{c}
f_{i} \\
r_{i} \times f_{i}
\end{array}\right)
$$

where $r_{i}$ denotes the position vector of the $i-t h$ grasp point in the object coordinate frame origined at the center of mass.

Substituting (2) into (3) provides:

$$
\underline{w}_{i}=\sum_{j=1}^{m} \lambda_{i j} u_{i j}
$$

where:

$$
u_{i j}=\left(\begin{array}{c}
l_{i j} \\
r_{i} \times l_{i j}
\end{array}\right)
$$

We normalize vectors $u_{i j}$ as follows:

$$
w_{i j}=\frac{1}{\left\|l_{i j}\right\|} u_{i j}
$$

The term $\left\|l_{i j}\right\|$ denotes the $L_{2}$ norm of vector $l_{i j}$. Vectors $w_{i j}$ are called primitive contact wrenches. Thus, $N=m n$ is the total number of primitve contact wrenches applied at the object by $n$ fingers.

Definition 6: The wrench matrix, $W$, is a $6 \times n m$ matrix where its column vectors are the primitive contact wrenches.

$$
\mathbf{W}=\left(\begin{array}{ccccc}
l_{11} & \ldots & l_{16} & \ldots & l_{n m} \\
r_{1} \times l_{11} & \ldots & r_{1} \times l_{16} & \ldots & r_{n} \times l_{n m}
\end{array}\right)
$$

\section{B. Force-Closure Preliminaries}

The stability of a grasp is characterized by force-closure property. This paragraph presents a definition of this property and an important result (proposition 3). This proposition is used, in section 3 , to formulate a frictional force-closure test.

Definition 7: According to the definition of Salisbury [2], a grasp has force-closure if and only if any external wrench can be balanced by the wrenches at the fingertips.

Proposition 1: A necessary and sufficient condition for force-closure is that the primitive contact wrenches resulted by contact forces at the contact points positively span the entire 6-dimensional wrench space.

Proof. for a proof, the reader should refer to [2].

Definition 8: A set of vectors , $\left\{v_{i}\right\}$, positively span a vector space if any vector $v$ in this space can be written as a positive linear combination of $v_{i}$, namely :

$$
v=\sum_{i \in I} \alpha_{i} v_{i}, \quad \alpha_{i} \geq 0
$$


Proposition 2: For any n-dimensional Euclidean space $\mathrm{E}^{\mathrm{n}}$, $n+1$ vectors are necessary to positively span $\mathrm{E}^{\mathrm{n}}$.

Proof. for a proof, the reader should refer to the relative linear algebra results presented by Goldman and Tucker [13].

Lemma 1: Given a set of $n+1$ vectors, $v_{1}, v_{2}, \ldots, v_{n+1}$, in $R^{n}$, such that $v_{1}, v_{2}, \ldots, v_{n}$ are linearly independent and :

$$
v_{n+1}=\sum_{i=1}^{n} \alpha_{i} v_{i}, \quad \alpha_{i}<0
$$

Then each $v_{i}, i=1, \ldots, n+1$, is a unique negative linear combination of the other $n$ vectors [14].

Proof. It is obviously true for $v_{n+1}$. for any $v_{i}, \mathrm{i}=1, \ldots, \mathrm{n}$, if we solve (8) for $v_{i}$, we have:

$$
v_{i}=\frac{1}{\alpha_{i}} v_{n+1}-\sum_{j=1, j \neq i}^{n+1} \frac{\alpha_{j}}{\alpha_{i}} v_{j}
$$

It is clear by (9) that $v_{i}, \mathrm{i}=1, \ldots, \mathrm{n}$, is a unique negative linear combination of the other $n$ vectors.

Proposition 3: A set of $n+1$ vectors $v_{1}, v_{2}, \ldots ., v_{n+1}$ in $R^{n}$ positively span $E^{n}$ if and only if $v_{n+1}$ is a unique linear combination of $v_{i}, i=1, \ldots, n$ and all coefficients are strictly negative [14].

Proof. for a proof, the reader should refer to [14]

\section{Grassmann Algebra Preliminaries}

As $3 D$ force-closure grasps involve $6 D$ wrench space. With a mere change of mathematical representation, using Grassmann algebra, we prove that wrenches, associated to any three non-aligned contact points of 3D objects, form a basis of the $6 D$ wrench space.

Plücker coordinates: Let $L$ be a line in the $3 D$ space. Let $u$ be the unit line direction and $P$ a point chosen on $L$. The direction vector along with its cross product with $P$ are known as Plücker coordinates and are denoted by $(u ; P \times u)$. These 6 coordinates represent $L$ in $3 D$ space [15], [16]. Consequently a primitive contact wrench, defined as $w_{i}=\left(f_{i} ; r_{i} \times f_{i}\right)$ can also be seen as a representation of the line of action $L_{f i}$ of the force $f_{i}$ applied at the point $r_{i}$. The 6 coordinates $\left(w_{i 1}, w_{i 2}, \ldots, w_{i 6}\right)$ of $w_{i}$ are called the Plücker coordinates of the line of action of $f$.

The Plücker coordinates are homogenous coordinates for a projective space of dimension $5, P^{5}$ : the wrenches $w_{i}$ and $\lambda w_{i}$, with $\lambda \neq 0$ both represent the same line $L_{f i}$. Then every line $L_{f i}$ in the $3 D$ space corresponds exactly to one point in $P^{5}$. The set of lines form a quadric, called the Grassmannian, defined by $w_{1} w_{4}+w_{2} w_{5}+w_{3} w_{6}=0$ in this projective space. At this point, we have defined a one-to-one relation between the set of lines in the $3 D$ space and points in $P^{5}$. The rank of this mapping is 6 .

Grassmann algebra : Grassmann studied manifold of lines which rank ranges varies from 0 to 6 . The purpose of this study was to find geometric characterization of each variety. We are going to use two main results of this study. For a proof of these results, the reader should refer to [17].

Proposition 4: All lines through one point are of rank 3.

Proposition 5: When all lines meet one special line, they are of rank 5 .

\section{FOUR FINGER FORCE-CLOSURE GRASPS}

At this point, we showed that a $6 D$ contact wrench can be represented by the line of action of its corresponding force. We use this mapping to prove that wrenches associated to three non-aligned contact points are of rank 6 . This result induces the formulation of a sufficient condition for four finger force-closure grasps.

Proposition 6: Wrenches associated to 3 aligned contact points are at most of rank 5 .

Proof. A $6 D$ contact wrench can be represented by the line of action of its corresponding force. The lines of action of forces applied at a contact point pass through that point. Thus wrenches associated to 3 aligned contact points meet one line, the one joining the 3 contact points. Consequently, from proposition 5 , these wrenches are at most of rank 5 .

Proposition 7: The 6 lines on the sides of a tetrahedron are independent, and thus form a $6 D$ basis, (Fig. 2).

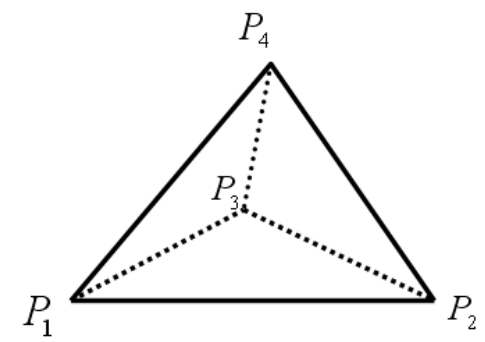

Fig. 2. The 6 lines of a tetrahedron are independent.

Proof. To deal with lines in $3 D$-space, we need a 4 dimensional linear space. For a basis of this space we can either take a point, $O$ and 3 vectors $e_{1}, e_{2}, e_{3}$ or 4 points $\left(p_{0}, p_{1}, p_{2}, p_{3}\right)$. We can relate these by:

$$
p_{1}=O ; p_{2}=O+e_{1} ; p_{3}=O+e_{2} ; p_{4}=O+e_{3}
$$

Any point can be written as a linear combination of these 4 points, for example:

$$
\begin{aligned}
& P_{a}=a_{1} p_{1}+a_{2} p_{2}+a_{3} p_{3}+a_{4} p_{4} \\
& P_{b}=b_{1} p_{1}+b_{2} p_{2}+b_{3} p_{3}+b_{4} p_{4}
\end{aligned}
$$


where the $a_{i}$ and $b_{i}$ are scalars and the sums of the $a_{i}$ and $b_{i}$ are unity.

Lines are represented in Grassmannian terms by exterior products of points. Hence from these 4 independent basis points we can construct 6 independent lines which intersect to form a tetrahedron :

$$
\begin{aligned}
& L_{1}=p_{1} \wedge p_{2} ; L_{2}=p_{1} \wedge p_{3} ; L_{3}=p_{1} \wedge p_{4} \\
& L_{4}=p_{2} \wedge p_{3} ; L_{5}=p_{2} \wedge p_{4} ; L_{6}=p_{3} \wedge p_{4}
\end{aligned}
$$

Any line is now able to be represented as a linear combination of these 6 basis lines. We can explicitly display this by multiplying out and simplifying the exterior product of two points on a chosen line:

$$
\begin{aligned}
& L=P_{a} \wedge P_{b}=\left(a_{1} p_{1}+a_{2} p_{2}+a_{3} p_{3}+a_{4} p_{4}\right) \wedge \\
& \left(b_{1} p_{1}+b_{2} p_{2}+b_{3} p_{3}+b_{4} p_{4}\right) .
\end{aligned}
$$

Proposition 8: Wrenches associated to 3 non-aligned contact points are of rank 6 .

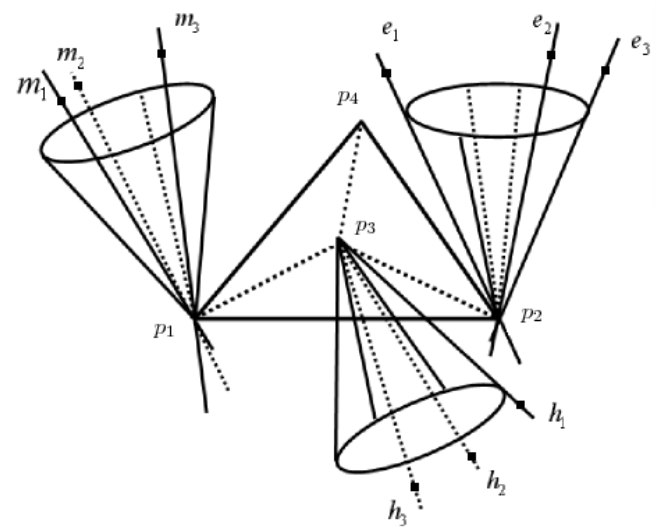

Fig. 3. The wrenches of rank 3 associated to the frictional contact points $p_{1}, p_{2}$ and $p_{3}$.

Proof. Let $p_{1}, p_{2}$ and $p_{3}$ be 3 non-aligned contact points. Consider the friction cone associated to $p_{1}$, called $C P_{1}$ (Fig. 3). Let $\left\{m_{1}, m_{2}, m_{3}\right\}$ be three points chosen on any 3 noncoplanar lines of this cone. The lines $\left\{l_{1}=p_{1} \wedge m_{1}, l_{2}=\right.$ $\left.p_{1} \wedge m_{2}, l_{3}=p_{1} \wedge m_{3}\right\}$ are of rank 3 , (from proposition 4 ). Thus any line that passes through $p_{1}$ can be expressed as a linear combination of these 3 lines. Similarly, $\left\{e_{1}, e_{2}, e_{3}\right\}$ and $\left\{h_{1}, h_{2}, h_{3}\right\}$, are associated respectively to the friction cones $C P_{2}, C P_{3}$ at $p_{2}, p_{3}$. In the same manner, $\left\{l_{4}=p_{2} \wedge e_{1}, l_{5}=\right.$ $\left.p_{2} \wedge e_{2}, l_{6}=p_{2} \wedge e_{3}\right\}$ and $\left\{l_{7}=p_{3} \wedge h_{1}, l_{8}=p_{3} \wedge h_{2}, l_{9}=\right.$ $\left.p_{3} \wedge h_{3}\right\}$ are either of rank 3 . Let $p_{4}$ be a point non-coplanar with $p_{1}, p_{2}, p_{3}$, so these 4 points constitute a tetrahedron.

The lines $\left(p_{1} \wedge p_{2}\right),\left(p_{1} \wedge p_{3}\right)$ and $\left(p_{1} \wedge p_{4}\right)$ can be expressed as a linear combination of $\left\{p_{1} \wedge m_{1}, p_{1} \wedge m_{2}, p_{1} \wedge m_{3}\right\}$ since they all pass through $p_{1}$, thus:

$$
\begin{aligned}
& p_{1} \wedge p_{2}=\sum_{i=1}^{3} \alpha_{i}\left(p_{1} \wedge m_{i}\right)=\sum_{i=1}^{3} \alpha_{i} l_{i} \\
& p_{1} \wedge p_{3}=\sum_{i=1}^{3} \beta_{i}\left(p_{1} \wedge m_{i}\right)=\sum_{i=1}^{3} \beta_{i} l_{i} \\
& p_{1} \wedge p_{4}=\sum_{i=1}^{3} \gamma_{i}\left(p_{1} \wedge m_{i}\right)=\sum_{i=1}^{3} \gamma_{i} l_{i}
\end{aligned}
$$

In the same manner, the lines $\left(p_{2} \wedge p_{3}\right)$ and $\left(p_{2} \wedge p_{4}\right)$ can be expressed as a linear combinations of $\left\{p_{2} \wedge e_{1}, p_{2} \wedge e_{2}, p_{2} \wedge e_{3}\right\}$ since they pass through the contact point $p_{2}$. Finally the line $\left(p_{3} \wedge p_{4}\right)$ passes through $p_{3}$ and thus can be expressed as a linear combination of $\left\{p_{3} \wedge h_{1}, p_{3} \wedge h_{2}, p_{3} \wedge h_{3}\right\}$.

Since the lines of the tetrahedron are of rank 6 (from proposition 6), they form a basis of $R^{6}$. We showed that the lines of the tetrahedron can be expressed as a linear combination of the 9 lines $l_{i}$. Thus these 9 lines, associated to the 3 friction cones, are also of rank 6 . Consequently, a 6 -dimensional basis can be extracted from these 9 lines. We remind the reader that the choice of 3 lines among the $m$ sides of each linearized friction cone is due to the fact that these $m$ lines are of rank 3 (from proposition 4 ).

Proposition 9: Assume that the grasp of 3 non-aligned fingers is not force-closure. Suppose that $\left\{b_{i}\right\}$ is the 6dimensional basis associated to their corresponding contact wrenches. A sufficient condition for a 4-finger force-closure grasp is that there exists a contact wrench $\gamma$ such that:

$$
\begin{aligned}
& \text { - } \gamma \text { is inside the linearized friction cone } \\
& \text { of the 4th finger } \\
& \text { - } \gamma=\sum_{i=1}^{6} \beta_{i} b_{i}, \beta_{i}<0 \\
& \Rightarrow \gamma=B \beta \Rightarrow \beta=B^{-1} \gamma
\end{aligned}
$$

where $B=\left[b_{1}, b_{2}, \ldots, b_{6}\right]$ is a $6 \times 6$ matrix and $\beta=\left[\beta_{1}, \beta_{2}, \ldots, \beta_{6}\right]^{T}$ is a $6 \times 1$ strictly negative vector. Thus, a simple multiplication by $B^{-1}$ permits to test if a contact wrench $\gamma$, and consequently the location of the $4 t h$ contact point, ensures a force-closure grasp.

Proof. A necessary and sufficient condition for forceclosure is that the primitive contact wrenches resulted by contact forces at the contact points positively span the entire 6-dimensional wrench space, (from proposition 1 ). A set of 7 vectors in $R^{6}$ positively span $E^{6}$ if and only if the seventh vector is a unique linear combination of the other six vectors and all coefficients are strictly negative, (from proposition 3). The seven vectors $\left\{\gamma, b_{i}, i=1, . .6\right\}$ satisfy these conditions 
TABLE I

COMPUTING 4-FINGER FORCE-CLOSURE GRASPS RESULTS

\section{N-FINGER FORCE-CLOSURE GRASPS SYNTHESIS}

We presented, in proposition 9, a sufficient condition for four-finger force-closure grasps. This condition can be easily applied to $n$-finger grasps $(n \geq 4)$.

To achieve force-closure, the grasp matrix should positively span the wrench space (proposition 1). Our method generates first, randomly, locations of $n-1$ non-aligned fingers. We showed that wrenches associated to 3 non-aligned contact points are of rank 6 (proposition 8). Thus, we find then all 6 dimensional basis from the wrenches associated to these $n-1$ contacts. A limited number of basis is selected. A position of the nth finger is located such that an associated contact wrench can be uniquely expressed as a strictly negative linear combination of one of the basis (proposition 9).

\section{A. The proposed approach}

This paragraph details the different steps of the algorithm computing force closure grasps of a $3 D$ object.

\section{Algorithm}

1. Input: - points representing a 3D object

2. - linearized friction cone at each point

$3 . \quad$ and corresponding wrenches

4. Output: - All possible locations of the $n^{\text {th }}$ finger

5. BEGIN

6. Rand_Fingers (n-1)

7. basis $=$ Find_Basis (wrenches)

8. rbasis $=$ Rand_Basis (basis)

9. for all object vertices

10. if Force_Closure (vertex, rbasis)

$11 . \quad$ add_vertex_solution (vertexList)

12.

13. return (vertexList)

14. END

Given a $3 D$ representation of an object along with normal directions and a friction coefficient, wrenches associated to each of its vertices are firstly computed. In order to obtain $n$-finger force-closure grasps, the function Rand_Fingers generates randomly, locations of $n-1$ non-aligned fingers on the object surface. All 6-dimensional basis from the wrenches associated to these $n-1$ contacts are determined by Find_Basis. A limited number of basis, rbasis, is then randomly selected using Rand_Basis function. Finally, all object vertices are tested for a $n$-finger force-closure grasp with Force_Closure. A position of the nth finger is located such that an associated contact wrench can be uniquely expressed as a strictly negative linear combination of one of the basis. We choose the wrench associated to the normal force on the $n t h$ contact. If the grasp ensures force-closure, add_vertex_solution stores the corresponding vertex in a vertexList. The latter contains all the possible locations of the nth finger ensuring force-closure grasps.

\begin{tabular}{|c||c||c||c||c||c||c||}
\hline \multicolumn{1}{|c||}{} & \multicolumn{3}{c|}{ number of Solutions } & \multicolumn{3}{c|}{ Time (s) } \\
\hline & classic & new & ratio & classic & new & ratio \\
\hline (a) & 210 & 35 & $16.7 \%$ & 2.483 & 0.697 & $28 \%$ \\
\hline (b) & 372 & 261 & $70 \%$ & 3.556 & 0.719 & $20 \%$ \\
\hline (c) & 566 & 371 & $65 \%$ & 5.1 & 1.02 & $20 \%$ \\
\hline (d) & 409 & 223 & $54.5 \%$ & 4.78 & 1.07 & $22.4 \%$ \\
\hline average & & & $51.5 \%$ & & & $22.6 \%$ \\
\hline
\end{tabular}

\section{EXPERIMENTAL RESULTS}

Our method sacrifices completeness in favor of fast computation. The obvious question is how it competes with a complete method. We choose to use in our experiments the classical complete method based on the construction of a $6 \mathrm{D}$ convex hull [21]. The process involves approximating the contact friction cones as a convex sum of a finite number of force vectors around the boundary of the cone, computing the associated object wrench for each force vector, and then finding the convex hull of this set of wrenches. If the origin is contained within this space, the grasp have force-closure. Otherwise, there exists some set of disturbance wrenches that cannot be resisted by the grasp.

We accomplish tests on four 3D object models, shown in (Fig. 4) represented by their vertices and their respective normal directions.

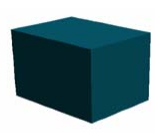

(a)

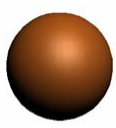

(b)

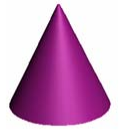

(c)

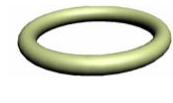

(d)
Fig. 4. 4 objects chosen as a testbed for our approach.

The two methods require the cone to be linearized. We use a 6-sided pyramid to represent a linear model of a cone. The test consists of randomly generating non-aligned locations of 3 fingers not in force-closure on each model. All vertices of the model are then tested to see if they ensure a four-finger force-closure grasp. With a 6-sided pyramid, the number of basis computed from the wrenches associated to the 3 fingers is approximately of 18000 . We randomly choose 100 basis and test the model vertices for each of these basis. Let $n$ be the number of vertices of a model, thus the time obtained with our method is for $100 \times n$ force-closure tests and the one obtained with the classical method is for $n$ tests. We repeat this procedure 10 times for each model. The result of experiments are shown in table (I). Our method is labelled as "new" and the convex hull method as "classic".

The experiments were run on Pentium Core duo machine with $2 G B$ memory and a CPU at $2.13 \mathrm{GHz}$. The program is implemented in $\mathrm{C}++$. 


\section{DISCUSSION}

The force-closure test we propose is sufficient but not necessary. In other words, our method reports many fault negative results (the method implies no force-closure when it exists). That is due to two reasons. The first one is the linearization of the friction cone, the second is that a point is not tested for a force-closure with all basis.

The first two columns, in (I), show the number of force-closure grasps found for each model. The fourth and the fifth columns show the corresponding computation time. We should mention that the time of computation varies between the different examples according to the number of their constituting vertices. The box, for example, is constituted of 602 vertices. As we randomly generate 10 times locations of 3 non-aligned fingers, 210 and 35 are respectively the average number of solution vertices found with the classical method and with ours. The computation time are respectively $2.483 \mathrm{~s}$ and $0.697 \mathrm{~s}$. Thus, as we test force-closure for 100 basis, $0.697 /(100 \times 602)$ and $2.483 / 602$, which are $1.15 e-5 s$ and $4.1 e-3 s$, are respectively the force-closure test time of our method and that of the classical method. Similarly, the tore is constituted of 1225 vertices. Thus, $1.07 /(1225 \times 100)=8.73 e-006 s$ and $4.78 / 1225=3.9 e-3 s$ are the corresponding force-closure test computation time. It is clear that our method is much faster but less complete. It reports many fault negative results. That is why we compute the ratio of the number of force-closure grasps found and the ratio of computation time. According to table (I), our method can find approximately $51.5 \%$ of the total number of solutions with a $22.6 \%$ time. In other words, it is half complete with the fifth computing time of the complete method.

We believe that the computation time of our method can still be improved by defining a criteria allowing the choice of good basis instead of selecting them randomly.

\section{CONCLUSIONS}

Computation procedures of force-closure grasps are crucial to solve dextrous manipulation planning problems. Simplifying these procedures is an important contribution for robots to autonomously manipulate objects. Toward this end, we presented an efficient algorithm for computing $n$-finger force-closure grasps. We used Grassmann algebra to prove that wrenches associated to any three non-aligned contact points of $3 D$ objects, form a basis of the 6-dimensional wrench space. Thus, given locations of $n-1$ fingers, not in force-closure, our algorithm is able to find wrench space basis associated to these contact points. It finds then all possible locations of the $n t h$ finger. A wrench associated to the $n t h$ finger is tested for ensuring $n$-finger force-closure by a simple inverse basis matrix multiplication.

Our method is general, it can be applied with any number $n \geq 4$ of fingers. The robot can chooses randomly the locations of the $n-1$ fingers on the object surface, with the only condition of the non-linearity of these contact points.
Then, it finds the position of the $n t h$ finger.

\section{ACKNOWLEDGMENTS}

I would like to thank John Browne for the insightful discussions. Thanks also to Maurice Milgram and Siohoi Ieng for suggesting some improvements to this paper.

\section{REFERENCES}

[1] A. Bicchi, On the closure properties of robotic grasping, Int. J. Robot. Res., vol. 14, no. 4, pp. 319-334, 1995.

[2] J.K. Salisbury and B. Roth, Kinematic and force analysis of articulated hands, ASME J. Mech., Transmissions, Automat., Design, vol. 105, pp. 33-41, 1982.

[3] Y.H. Liu, Qualitative test and force optimization of 3-D frictional form closure grasps using linear programming, IEEE Transactions on Robotics and Automation, vol. 15, no. 1, 1999.

[4] B. Mishra, J.T. Schwartz, and M. Sharir, On the existence and synthesis of multifinger positive grips, Algorithmica, Special Issue: Robotics, vol. 2, pp. 541-558, 1987.

[5] D.J. Montana, The condition for contact grasp stability, in Proc. IEEE Int. Conf. Robot. Automat., pp. 412-417, 1991.

[6] R.M. Murray, Z. Li, and S.S. Sastry, A Mathematical introduction to robotic manipulation, Orlando, FL: CRC, 1994.

[7] B. Mirtich and J. Canny, Easily Computable Optimum Grasps in 2-D and 3-D, Proc. IEEE Int. Conf. Robot. Automat., vol. 1, pp. 739-747, 1994.

[8] D. Ding, Y.H. Liu, and S. Wang, Computing 3D optimal form-closure grasps, Proc. IEEE Int. Conf. Robot. Automat., 2000.

[9] J.C. Trinkle, "On the stability and instantaneous velocity of grasped frictionless objects", IEEE Trans. Robot. Automat., vol. 8, pp. 560-572, 1992.

[10] J.C. Trinkle, "A quantitative test for form-closure grasps", Proc. IEEE Int. Conf. Intell. Robots Syst., pp. 1670-1677, 1992.

[11] F. Reuleaux, "The kinematics of machinery", Macmillanv and company, 1876.

[12] V.D. Nguyen, "Constructing force-closure grasps", Int. J. Robot. Res., vol. 7, no. 3, pp. 3-16, 1988.

[13] A.J. Goldman and A.W. Tucker, "Polyhedral Convex Cones", Princeton University Press, 1956.

[14] R. Wagner, Y. Zhuang and K. Goldberg, "Fixturing faceted parts with seven modular struts", IEEE International Symposium on Assembly and Task Planning, USA, 1995.

[15] H. Crapo, "A combinatorial perspective on algebraic geometry", Colloquio Int. sulle Teorie Combinatorie, Roma, 1973.

[16] O. Veblen, J.W. Young, "Projective geometry", the Athenaeum press, 1910.

[17] A. Dandurand, "The rigidity of compound spatial grid", Structural topology 10, 1984.

[18] Ch. Borst, M. Fischer and G. Hirzinger, "Grasping the dice by dicing the grasp", IEEE Int. Conf. on Intelligent Robots and Systems, 2003.

[19] N. Niparnan and A. Sudsang, "Positive span of force and torque components of four-fingered three-dimensional force-closure grasps", IEEE Intl. Conf. on Robotics and Automation, 2007.

[20] X. Zhu and J. Wang, "Synthesis of Force-Closure Grasps on 3D Objects Based on the Q Distance", IEEE Transcations on robotics and Automation, vol. 19 , no. $4,2003$.

[21] A.T. Miller and P.K. Allen, "Examples of 3D grasp quality computations", Proc. IEEE Intl. Conf. on Robotics and Automation, pp. 12401246, 1999.

[22] A. Bicchi, "Hands for dexterous manipulation and robust grasping: A diffcult mad toward simplicity,", IEEE Trans. on Robotics and Autornorion, vol. 16 , no. 6, pp. 652-662, 2000.

[23] P. R. Kraus, V. I. Kumar, and P. Dupont. "Analysis of frictional contact models for dynamic symulation." in IEEE Intl. Conf. on Robotics and Automation, 1997. 